\title{
Understanding mental health difficulties and associated psychosocial outcomes in adolescents in the HIV clinic at Kenyatta National Hospital, Kenya
}

\author{
Douglas Gaitho ${ }^{1 *}$, Manasi Kumar ${ }^{2}$, Dalton Wamalwa' ${ }^{1}$, Grace Nduku Wambua ${ }^{2}$ and Ruth Nduati ${ }^{1}$
}

\begin{abstract}
Background: Globally adolescents continue to have an upward trend in HIV incidence and AIDS-related mortality. The interplay between the rapid physical growth, sexual maturation, and enormous albeit slow-evolving cognitive and psychological changes in adolescence may partly explain this trend. Our main purpose was to highlight key psychosocial characteristics of HIV-infected adolescents and explore if these characteristics are associated with depression symptoms.
\end{abstract}

Methods: From August to December 2016 after obtaining informed consent, adolescents living with HIV at Kenyatta National Hospital were interviewed using the Home environment, Education and Employment, Activity, Sexuality, Suicide and depression traits (HEADSS) tool combined with the Patient Health Questionnaire (PHQ-9) to elucidate which key symptoms of depression and link with psychosocial characteristics mapped on HEADSS. In order to determine which psychosocial characteristics were linked with risk of depression, the traits of adolescents who were symptomatic were compared to those who were not using univariate and multivariate regression analysis.

Results: All the 270 adolescents offered participation in the study accepted to enroll. The aged 10-19 years were recruited and mean age was 14.75 and 53.7\% $(n=145)$ were males. Overall, $269(99.9 \%)$ were still in school and 52.6\% of the adolescents had symptoms of depression. The independent predictors of depression were being of ages $15-19$ years $[\mathrm{OR}=2.34(95 \% \mathrm{Cl} 1.36,4.04) P<0.02]$, ever repeating classes $[\mathrm{OR}=1.74(95 \% \mathrm{Cl} 1.0-3.05) P=0.05]$, ever being sent away from school due to lack of school fees $[\mathrm{OR}=1.71(95 \% \mathrm{Cl} 1.0-2.91) P=0.05]$, and non-adherence to medication $[\mathrm{OR}=1.84(95 \% \mathrm{Cl} 1.08-3.14) P=0.03$. Missing of meals due to food insecurity was associated with an important trend towards increased risk of depression $[O R=2.42(C l 0.96-6.14) P=0.06]$.

Conclusion: One in two of the adolescents interviewed had depression symptoms which were significantly associated with lack of school fees, missing meals, non-adherence to medication, and substance abuse.

Keywords: Adolescents, HIV, Psychosocial characteristics, Depression, School performance, Adherence

\footnotetext{
*Correspondence: dgaitho@gmail.com

${ }^{1}$ Department of Paediatrics, College of Health Sciences, University

of Nairobi, P.O. Box 19676, Nairobi, Kenya

Full list of author information is available at the end of the article
} 


\section{Background}

Of the 2.1 million HIV-infected adolescents, $83 \%$ of these reside in sub-Saharan Africa [1]. In 2013, for the first time age-disaggregated estimated numbers of adolescents living with HIV (ALHIV) were presented in a global report that estimated 250,000 new HIV infections and 120,000 AIDS-related deaths among adolescents in that year [1]. Further to this, adolescents were the only age group in which AIDS-related deaths had not decreased in the period from 2005 to 2013 contrasting sharply with the reported cumulative $38 \%$ decline in all the other age groups [2]. An additional observation was the very low scale up and treatment of ALHIV.

The global All In campaign to end adolescent AIDS was officially launched in Kenya in February 2015 [2]. Kenya then released a Fast Track Plan to End Adolescent AIDS in September of the same year [3].

Implicit in the planned response is the assumption that adolescents will be able to take charge of their health care and all interventions would be multisectoral. Additionally, there is a tendency to lump all adolescents together failing to take into consideration that adolescence is a period of rapid growth and that there is significant lapse in time between sexual maturation taking place in adolescence (10-14 years) and/or emotional and cognitive maturation that takes place in late adolescence (17-24 years). This period of human development has well-described complex challenges that the HIV-infected adolescents have to get attuned to in addition to the problems related to their illness. Given that mental health issues are an emerging public health priority with suicide ranked as third highest cause of death among adolescents globally [4], ALHIV have higher prevalence rates of depression, anxiety, conduct, and functional disorders compared to HIV-unaffected adolescents [5-7]. HIV-related psychosocial problems include stigma and discrimination, relationship challenges such as HIV status disclosure, difficulties with their medication, loss of biological parents in cases of orphans, denial and ambivalence towards HIV, and school performance [8-10]. These have a major impact in the quality of life and if not adequately addressed can lead to poor outcomes [11]. These psychosocial adversities impact negatively on adherence to combined antiretroviral (cARV) medication [12, 13] and predispose adolescent greater depression risk. Risk factors such as being female, fewer years of schooling, death within the household, poor school performance, being in a relationship with the opposite sex, non-disclosure of HIV status, severe immunosuppression, and bullying in school for taking medication have been shown to be associated with depression [14].

Adolescence and especially mid-adolescence is characterized by increased exploration of one's environments and feelings of invincibility. In the process, a significant number of adolescents engage in risky behaviors including high-risk sexual encounters that expose them to sexually transmitted infections and unplanned pregnancies [Ndugwa et al., Patel et al.]. Experimentation with psychoactive agents such as alcohol and others may lead to addiction and fuel poor sexual-decision making. ALHIV have the additional stress of putting their partner at risk, challenges with disclosure of HIV status, and adverse drug reactions from the psychoactive agents or with use of hormonal contraceptive $[14,15]$.

The purpose of this study was to highlight key psychosocial characteristics of HIV-infected adolescents and explore if these characteristics predict depression. The WHO AA-HA 2017 recommends competencies and intervention implementation targeting different developmental age groups and using HEADSS framework as a guidance tool. We feel our work aligns closely to the WHO adolescent care framework [4], as understanding and describing these psychosocial characteristics of HIV-infected adolescents will aid in design and implementation of treatment and follow-up programs that are tailor-made for their needs, thereby reversing the negative trends in this age group (notably these are reducing risky sexual behavior and consequently reducing new HIV infections, addressing issues pertaining disclosure, adherence to medication, and stigma/discrimination).

\section{Methods \\ Study design}

This was a cross-sectional descriptive survey among HIV-infected adolescents, attending a Comprehensive Care Clinic at Kenyatta National Hospital in Nairobi. The research team developed a semi-structured questionnaire based on the HEADSS framework. The tool screened information about the home environment, involvement in exercise and activity, school performance, use of drugs and substances of abuse, involvement in sexual activity, and depression/suicide. The screen for depression used questions obtained from the Patient Health Questionnaire (PHQ-9) which was administered to all adolescents. The HEADSS assessment tool has been validated in Outpatient Departments in the United States of America and found to accurately screen for mental health problems (MHP) with reported sensitivity of $82 \%$ and a specificity of $87 \%$ in predicting psychiatric consult and admission to in-patient psychiatry [16]. It has, however, not been validated in our setting. It is for this reason we did not use a cut-off.

\section{Study variables}

The specific variables in the various domains of sociodemographic characteristics, Home environment, 
Education, Activity and routine, Drugs, Sexuality, Suicide and depression, Adherence to ARV drugs, and full disclosure of HIV status are shown in Table 1.

\section{Study population and setting}

Adolescents were eligible for the study if they were aged 10-19 years. The study was carried out between August and December 2016. Ethical approval was obtained from The Ethics and Research Committee of Kenyatta National Hospital and University of Nairobi (approval no. P217/03/2016). All 270 participants who were approached consented to participate.

\section{Study procedure}

Adolescents were offered study participation consecutively on a day they had attended a regularly scheduled clinic appointment. Written informed consent and assent was obtained from the caregiver and the adolescent, respectively. After this, the caregiver was interviewed first in the absence of the adolescent, and then the adolescent was interviewed on the expanded survey. The interview was carried out by the first and fourth authors according to the guidance of a senior psychiatrist consultant. The interview process lasted approximately half an hour. Those who experienced distress or had high PHQ-9 scores were referred to a psychiatrist immediately and received further psychosocial support at the clinic.

Study sample size was determined using Fischer's Formula from previously published estimates of depression. Kim et al. [14] found the prevalence of depression among ALHIV to be $18.9 \%$. By this token, our study was adequately powered to determine prevalence of depression.

\section{Data analysis}

The data were collected on a paper tool and then entered onto SPSS. Descriptive statistics was computed for the study population, continuous variables, and frequency and proportions for categorical variables were determined. Univariate and multivariate linear regression analysis was carried out to determine factors associated with depression. In the univariate analysis study, participants were characterized as having depression versus no depression. Chi-square statistics were used to determine whether there was an association between depression and the various participant variables collected using the HEADSS framework. Variables that had a $\mathrm{p}$ value of $<0.05$ in univariate analysis were entered into a multivariate linear regression to determine the independent predictors of depression. A Pearson's correlation analysis was carried out to safe-guard against collinearity. In this analysis, depression was treated as a continuous variable so as to increase the power of the study to find associations. We have reported beta values, odds ratios, and the $95 \%$ confidence intervals.

\section{Results}

Table 1 depicts the key characteristics of the study participants. The mean age of our sample of 270 was 14.75 years $(\mathrm{SD}=2.6$ years). This included 125 adolescents aged 10-14 years and 145 aged 15-19 years, of whom 145 (53.7\%) were male and 125 (47.3\%) female participants (Table 2).

Dividing key findings on the HEADSS framework, the following results are notable.

Home environment Eleven (4.1\%) study participants reported that they had encountered physical violence in their home, 18 (6.7\%) reported running away from home at least once, and another 59 (21.9\%) adolescents reported that they had changed homes in the past 1 year. In the past 2 weeks preceding the study, $80(29.6 \%)$ reported they had involuntarily missed a meal.

\section{Table 1 Psychosocial characteristics evaluated using HEADSS framework}

\footnotetext{
1. Socio-demographic characteristics — age, sex, education level, relation to the caregiver

2. Home environment - relationships with other household members, changing homes in the last 1 year, missing meals, and physical violence

3. Education — school performance, bullying, repeating classes, inability to pay school fees, change of schools in the last 2 years, and reasons for missing school

4. Activity and routine -involvement in extracurricular activities and religious activities

5. Drugs - use of drugs and substances of abuse

6. Sex-involvement in sexual activity, practice of safe sex, and worry about pregnancy/sexually transmitted diseases. Questions on sexual activity were restricted to adolescents aged 12 years and above

7. Suicide and depression - Any adolescent with a score of 1 or more on PHQ-9 was considered to have a depressive symptom

8. Adherence to ARV drugs — any positive response on any of the following 5 questions_do you ever refuse or miss drugs, don't take drugs in front of others, or have problems taking drugs daily or on time - was taken to be an indication of non-adherence

9. Full disclosure of HIV status - an adolescent was classified as having full disclosure if they listed HIV in responding to any of the following 3 questions why are you visiting the clinic, due to which illness, and why are you taking medication?
} 
Table 2 Factors associated with depression symptoms among HIV-infected adolescents on cARV and long-term follow-up at Kenyatta National Hospital

\begin{tabular}{|c|c|c|c|c|c|}
\hline \multirow[t]{2}{*}{ Parameter } & \multirow[t]{2}{*}{ Overall $(N=270)$} & \multicolumn{2}{|c|}{ Depression symptoms } & \multirow[t]{2}{*}{ OR $(95 \% \mathrm{Cl})$} & \multirow[t]{2}{*}{ Chi square ( $P$ value) } \\
\hline & & No $(n=128)$ & Yes $(n=142)$ & & \\
\hline \multicolumn{6}{|l|}{ Gender } \\
\hline Male & $145(53.7)$ & $70(54.7)$ & $77(54.2)$ & Ref. & \multirow[t]{2}{*}{$\left(x_{(1,270)}^{2}=0.01 ; P=0.939\right)$} \\
\hline Female & $125(46.3)$ & $58(45.3)$ & $65(45.8)$ & $1.0(0.6-1.7)$ & \\
\hline \multicolumn{6}{|l|}{ Age } \\
\hline $10-14$ & $125(46.3)$ & $75(58.6)$ & $50(35.2)$ & Ref. & \multirow[t]{2}{*}{$\left(X_{(1,270)}^{2}=14.80 ; P=0.001\right)$} \\
\hline $15-19$ & $145(53.7)$ & $53(41.4)$ & $92(64.8)$ & $2.60(1.6-4.3)$ & \\
\hline \multicolumn{6}{|l|}{ School environment } \\
\hline Happy with school performance & $193(71.5)$ & $92(71.9)$ & $101(71.1)$ & $0.96(0.6-1.6)$ & \multirow{7}{*}{$\begin{array}{l}\left(X_{(1,270)}^{2}=0.02 ; P=0.9\right) \\
\left(X_{(1,270)}^{2}=0.66 ; P=0.4\right) \\
\left(X^{2}{ }_{(1,270)}=0.72 ; P=0.4\right) \\
\left(X^{2}{ }_{(1,270)}=1.04 ; P=0.3\right) \\
\left(X^{2}{ }_{(1,270)}=3.67 ; P=0.05\right) \\
\left(X^{2}{ }_{(1,270)}=5.56 ; P=0.02\right) \\
\left(X_{(1,270)}^{2}=7.85 ; P=0.005\right)\end{array}$} \\
\hline Plan to continue with education & $260(96.3)$ & $122(95.3)$ & $138(97.2)$ & $1.70(0.5-6.2)$ & \\
\hline Been bullied in school & $50(18.5)$ & $21(16.4)$ & $29(20.4)$ & $1.31(0.7-2.4)$ & \\
\hline School performance dropping & $49(18.1)$ & $20(15.6)$ & $29(20.4)$ & $1.39(0.7-2.6)$ & \\
\hline Changed schools in the past 2 years & $85(31.5)$ & $33(25.8)$ & $52(36.6)$ & $1.66(1.0-2.8)$ & \\
\hline Ever repeated a class & $91(33.7)$ & $34(26.6)$ & $57(40.1)$ & $1.85(1.1-3.1)$ & \\
\hline Ever sent away from school due to lack of fees & $119(44.1)$ & $45(37.8)$ & $74(62.2)$ & $2.01(1.2-3.3)$ & \\
\hline \multicolumn{6}{|l|}{ Activity } \\
\hline Has extracurricular activities & $190(70.4)$ & $90(70.3)$ & $100(70.4)$ & $1.0(0.6-1.7)$ & \multirow{2}{*}{$\begin{array}{l}\left(X^{2}{ }_{(1,270)}=0.00 ; P=0.9\right) \\
\left(X^{2}(1,270)=0.23 ; P=0.6\right)\end{array}$} \\
\hline Attend religious activities & $251(93.0)$ & $120(93.8)$ & $131(92.3)$ & $1.26(0.5-3.2)$ & \\
\hline \multicolumn{6}{|l|}{ Home environment } \\
\hline Changed homes in the last 1 year & $59(21.9)$ & $28(21.9)$ & $31(21.8)$ & $1.00(0.6-1.8)$ & \multirow{4}{*}{$\begin{array}{l}\left(X^{2}{ }_{(1,270)}=0.00 ; P=0.99\right) \\
\left(X^{2}{ }_{(1,270)}=4.91 ; P=0.03\right) \\
\left(X^{2}{ }_{(1,270)}=0.02 ; P=0.895\right) \\
\left(X_{(1,270)}^{2}=6.84 ; P=0.009\right)\end{array}$} \\
\hline Ever ran away from home & $18(6.7)$ & $4(3.1)$ & $14(9.9)$ & $3.39(1.1-10.6)$ & \\
\hline Physical violence at home & $11(4.1)$ & $5(3.9)$ & $6(4.2)$ & $1.09(0.3-3.7)$ & \\
\hline Involuntarily misses meals because of lack of food & $34(12.6)$ & $9(7.0)$ & $25(17.6)$ & $2.83(1.3-6.3)$ & \\
\hline \multicolumn{6}{|l|}{ Drug and substance use } \\
\hline Family members or friends who use drugs & $96(35.6)$ & $41(32.0)$ & $55(38.7)$ & $0.75(0.5-1.2)$ & \multirow{2}{*}{$\begin{array}{l}\left(X_{(1,270)}^{2}=1.32 ; P=0.3\right) \\
\left(X_{(1,270)}^{2}=6.64 ; P=0.01\right)\end{array}$} \\
\hline Use drugs/substance abuse & $23(8.5)$ & $5(3.9)$ & $18(12.7)$ & $3.57(1.3-10.0)$ & \\
\hline \multicolumn{6}{|l|}{ Sexuality } \\
\hline Agree that condoms reduces HIV transmission & $126(46.7)$ & $52(40.6)$ & $74(52.1)$ & $1.59(1.0-2.6)$ & \multirow{3}{*}{$\begin{array}{l}\left(X^{2}(2,270)=3.83 ; P=0.1\right) \\
\left(X^{2}(1,228)=0.22 ; P=0.6\right) \\
\left(X_{(1,29)}^{2}=0.05 ; P=0.8\right)\end{array}$} \\
\hline Ever had sex $(N=228)$ & $31(13.6)$ & $12(12.4)$ & $19(14.5)$ & $1.20(0.6-2.6)$ & \\
\hline Inconsistently using condoms $(N=29)$ & $20(69.0)$ & $8(66.7)$ & $12(70.6)$ & $1.20(0.2-5.9)$ & \\
\hline \multicolumn{6}{|l|}{ HIV Disclosure } \\
\hline Full HIV disclosure & $174(64.4)$ & $78(60.9)$ & $96(67.6)$ & $1.34(0.8-2.2)$ & $\left(X_{(1,270)}^{2}=1.31 ; P=0.3\right)$ \\
\hline \multicolumn{6}{|l|}{ Adherence to medication } \\
\hline Non-perfect adherence to medication & $139(51.5)$ & $50(39.1)$ & $89(62.7)$ & $2.62(1.6-4.3)$ & \multirow[t]{2}{*}{$\left(X_{(1,270)}^{2}=15.02 ; P<0.001\right)$} \\
\hline Perfect adherence to mediation & $131(48.5)$ & $78(60.9)$ & $53(37.3)$ & Ref. & \\
\hline
\end{tabular}

Education Overall 269 (99.6\%) of the study participants were in school, 137 participants $(50.7 \%)$ were enrolled in primary school, 98 (36.3\%) in secondary school, and 35 (13\%) participants in tertiary or vocational training institutions. Overall, $260(96.3 \%)$ of the adolescents were committed to furthering their education. The commonest reason for ever missing school was sickness as reported by 265 (98.1\%) of our participants. Bullying in school was reported by $50(18.5 \%)$ study participants. A total of 91 (33.7\%) study participants had repeated a grade, 77 (29\%) participants reported dissatisfaction with their current school performance, and 49 (18.1\%) participants had experienced a drop in their grades. A total of 119 (44.1\%) participants had at least once been sent away from school due to lack of tuition fees.

Activity and routine Up to 190 (70.4\%) of the study participants were involved in extracurricular activities which included sports and other hobbies. Almost all of the study participants, 251 (93\%), attended religious activities. 
Drugs Only 23 (8.5\%) acknowledging use of drugs and substances of addiction, while 96 (35.6\%) reported use of the same among family or friends. Sexual activity There were 229 study participants aged 12 years and above who were interviewed regarding their sexual activity. Among this group 31 (13.6\%) reported they had achieved sexual debut, including 10 (35.7\%) participants for whom first sexual encounter was at 18 years of age. Overall, 19 (73\%) participants of the sexually active adolescents had one sexual partner. Of those who reported had ever had sex, 20 (64.5\%) participants reported inconsistent condom use. Sexually active adolescents obtained condoms from health facilities. Eight (3\%) adolescents reported having a forced sexual encounter. This means 8 of the 31 (26\%) sexual debuts may have been through a forced sexual encounter or abuse of some kind.

Suicide and depression Overall, 142 (52.6\%) had at least one symptom of depression. The mean depression score was $1.78(\mathrm{SD}=2.81)$. Twelve $(4.4 \%)$ of the adolescents were found to have suicidal ideation in the last 2 weeks preceding the survey. Suicidal adolescents were immediately referred to a psychiatrist, while the others were referred to a specialist counselor based within CCC and all these cases received psychiatric oversight.

Drug adherence Overall, 139 (51.5\%) of the study participants reported a non-perfect adherence to their medications.

\section{Univariate analyses of risk factors for depression}

Male and female adolescents had similar levels of depression symptoms. Adolescents aged 15-19 years had a significantly higher prevalence of depression symptoms compared to those aged 10-14 years, 92 (63.4\%) versus 50 (40\%) of our total sample [OR $=2.6$ (CI 1.6-4.3), $P<0.001)]$. Depression was significantly associated with frequent changing of schools in the preceding 2 years due to repeated adversities with $52(61.2 \%)$ of 142 versus $33(38.8 \%)$ of 128 participants, [OR $=1.66$ (95\% CI 0.992.81), $P=0.05$ ]. Depressed adolescents were significantly more likely to report ever repeating a grade as compared to those who were not depressed with 57 (62.6\%) of 142 versus $34(37.4 \%)$ of 128 [OR $=1.85$ (95\% CI $1.11-3.11)$, $P=0.02]$. A significantly higher proportion of adolescents with symptoms of depression reported that they had ever been sent back home for lack of school fees 74 (62.2\%) of 148 compared to 45 (37.8\%) of 128 , who had no symptoms $[\mathrm{OR}=2.01$ ( $95 \%$ CI $1.23-6.31), P=0.005]$.

In the home environment, 25 (18.3\%) of 142 adolescents experiencing symptoms of depression reported that they involuntarily missed meals due to unavailability of food compared to 9 (7\%) of 128 without such symptoms, $[\mathrm{OR}=2.83$ (95\% CI 1.27-6.31), $P=0.009]$. Adolescents who were depressed were more likely to report that they had at least once ran away from home 14 (9.9\%) versus 4 (3.1\%), $[\mathrm{OR}=3.39$ (95\% CI 1.09-10.58), $P=0.03$ ]. Adolescents who were depressed had a significantly higher likelihood of reporting substance use compared to those who had no symptoms of depression 18 (12.7\%) versus 5 (3.9\%), $[\mathrm{OR}=3.57$ (95\% CI 1.29-9.92), $P=0.01]$. Use of drugs by family members was not associated with symptoms of depression. Adolescents who had experienced any symptom of depression were significantly more likely to report non-perfect adherence to their medications 89 (62.7\%) versus 50 (39.1\%), [OR=2.62 (95\% CI 1.60, 4.28), $P \leq 0.001]$.

\section{Multivariate analysis of association between PHQ-9 scores and other variables}

The independent correlates of depression in the group of adolescents were being of ages $15-19$ years [OR $=2.34$ (95\% CI 1.36-4.04), $P<0.02$ ], having had an experience of repeating a grade $[\mathrm{OR}=1.74(95 \% \mathrm{CI} 1.0-3.05) P=0.05]$, having had an experience of being refused school participation due to lack of school fees $[\mathrm{OR}=1.71$ (95\% CI 1.0-2.91) $P=0.05]$, and non-adherence to medication $[\mathrm{OR}=1.84$ (95\% CI 1.08-3.14) $P=0.03$ ] (Table 3).

\section{Discussion}

This study is among the few in Kenya to comprehensively assess at the health facility various psychosocial issues that are faced by ALHIV. Of importance is that these issues have not become part of routine assessment and management during regular clinic visits and these psychosocial correlates in the literature have been strongly associated with depression. The HEADSS tool framework was adopted into the adolescent tool kit called Adolescent Package of Care (APOC) by the Kenya Ministry of health to support care of adolescents; however, the specific questionnaire has not been adapted into the Kenyan context yet [17]. The standard HEADSS tool was shortened and adapted to the Kenyan context and a standard depression screening tool added. The tool is included as supplementary study material. This is the first study to provide empirical evidence on the performance of this HEADSS framework in assessing ALHIV since this framework was adopted in APOC guidelines. As noted in our introduction, WHO AA-HA (2017) stipulates the use of HEADSS tool for not only needs assessment but as a framework for intervention development looking at different developmental competencies and domains in which adolescent development and health care needs have to be conceptualized [4]. 
Table 3 Correlates of depression symptoms

\begin{tabular}{|c|c|c|c|c|c|c|}
\hline \multirow[t]{2}{*}{ Parameter } & \multirow[t]{2}{*}{$\beta$} & \multirow[t]{2}{*}{ S.E } & \multirow[t]{2}{*}{ A.O.R } & \multicolumn{2}{|l|}{$95 \% \mathrm{Cl}$} & \multirow[t]{2}{*}{$P$ value } \\
\hline & & & & Lower & Upper & \\
\hline Age in years $15-19$ years & 0.85 & 0.28 & 2.34 & 1.4 & 4.0 & 0.02 \\
\hline Changed schools in the past 2 years & 0.39 & 0.29 & 1.47 & 0.8 & 2.6 & 0.2 \\
\hline Ever repeated a class & 0.56 & 0.28 & 1.74 & 1.0 & 3.0 & 0.05 \\
\hline Ever sent away from school due to lack of fees & 0.54 & 0.27 & 1.71 & 1.0 & 2.9 & 0.05 \\
\hline Ever ran away from home & 0.34 & 0.65 & 1.40 & 0.4 & 5.0 & 0.6 \\
\hline Involuntarily misses meals because of lack of food & 0.89 & 0.47 & 2.42 & 1.0 & 6.1 & 0.06 \\
\hline Use drugs/substance abuse & 0.55 & 0.58 & 1.72 & 0.6 & 5.4 & 0.3 \\
\hline Non-adherence to medication & 0.61 & 0.27 & 1.84 & 1.0 & 3.1 & 0.03 \\
\hline
\end{tabular}

In line with this, our work despite its shortcoming and narrow scope (being restricted to health facility) has high operational validity as we have directly explored with adolescents about their ongoing treatment and mental health needs. We have bifurcated analyses based on differential developmental competencies [18]. National adolescent priorities have been laid out in a number of complementary guidelines. The Kenyan national adolescent reproductive and sexual health policy [18], Vision 2030 [19], and UN SDGs such as goals focus on adolescent health. The Kenyan national policy focuses on adolescents' needs that have been specifically targeted such as reducing early sexual debut, knowledge about HIV transmission, and prevention in adolescent girls and boys and in adolescents of ages $10-14$ and $15-24$ years.

We evaluated 270 adolescents and a wide majority (99.6\%) as would be expected were still in formal education at different tiers. A study done by Souza et al. (2010) had a higher dropout rate, with $89.8 \%$ in school in the same population in Brazil [10]. Despite high numbers of those in formal education, the challenges came in the form of financial constraints that came in the way of parental capacity to pay for school fees in time. In our sample, $44.1 \%$ alluded to missing school due to unpaid school fees which prompted the school to discontinue these candidates. Additionally, there were also concerns about dropping school performance in $18.1 \%$ of our sample with $33.7 \%$ having repeated a grade and $18.5 \%$ having had difficult bullying experiences at school. In a study by Shavisa et al. [20], school dropout rates in the general population were 1.34 and $1.06 \%$ among boys and girls, respectively. The shame associated with poor performance and failure in tests may have exacerbated experiences of bullying. Bullying of vulnerable child who live in adversities also needs greater attention. A systematic review done found that problems with school functioning need early detection and intervention and prolonged problems may lead to long-term adjustment and psychological disorders [10].

Our study found older adolescents (age 15-19 years) with a twofold increased risk of depression. Adolescence is a period of significant cognitive and socio-emotional development [21]. Mental illness may manifest in a different way as these capacities develop. Given the complex pathogenesis of HIV illness, mental health problems, poor neurocognitive functioning, and psychosocial adversities in such vulnerable adolescents are subsets of an inter-related problem. Additionally, due to the HIV infection, these developing capacities are also compromised in ways that are also yet to be fully understood in both neurocognitive and psychosocial senses. Our crosssectional study design does not equip us sufficiently to extend this argument based on these results but the literature is replete with similar conclusions [12]. In this study, we used PHQ-9 to screen for depression symptoms and as such we did not find depression prevalence to be high, although we did find that $12(4.4 \%)$ participants reported suicidal ideation whom we referred for suicide watch and priority mental health care. The use of a standardized check list to screen for symptoms of depression was therefore a huge benefit for this set of study participants.

The other independent predictors for depression were repeating a class and poverty measured by the surrogate markers that included missing meals and being sent away from school due to inability to source school fees. There is emerging evidence from the National HIV program that school environment frequently destabilizes the adolescent leading to poor drug adherence [22]. In this study, we show two school-related factors that were associated, repeated changing of schools and being sent away due to lack of school fees, both factors connected to the material conditions of the families.

One in two of the adolescents in the study were not adherent to their medication. The measure of adherence 
in this study was a crude one in that it relied on selfreporting. A study by Vreeman et al. [23] comparing the use of Medication Events Monitoring System (MEMS) versus self-reported adherence demonstrated overreporting in the latter group. Further to this, non-adherence was one of the independent predictors of depression symptoms. In this cross-sectional study, we cannot reliably say what came first. There may be merit in offering psychotherapy immediately to ALHIV who have symptoms of depression as a preventative measure.

One in three adolescents live in an environment where they are exposed to drugs and other substances of abuse. One in ten of the adolescents interviewed reported substance abuse with alcohol being the most commonly used. The use of drugs increased the presence of depression symptoms by $70 \%$. These findings are very critical as related studies have found a strong association between mental health problems, risky sexual behaviors, and substance abuse [24] in both HIV- and non-HIV-infected adolescents. There should then be a deliberate effort to inform and counsel adolescents on the harms of drug and substance use. There is a need for further work on the tool to clarify whether the ALHIV really understand when we use the term 'drugs' in this context given the fact that they are on medication all the time.

The study found that involuntary missing of meals due to unavailability of food in the household was also associated with twofold increased risk of depression. People living with HIV are instructed to ensure that they do not miss meals as they take their cARV and we commonly find that people attribute non-adherence to paucity of food and to a fallacy that given the food insecurity, taking drugs without food would be harmful for the body. All these experiences probably increase the sense of vulnerability and stigma for the adolescent who lives among so many social, financial, and emotional adversities. This study did not collect formative data that would better explain the impact of these factors on the adolescents and we do feel this is an important area to invest in. A study carried out in Malawi by Kim et al. [14] found bullying because of being on medication, severe immunosuppression, and having a boyfriend/girlfriend was associated with depression. This study did not find any significant associations between depression symptoms and bullying and we did not collect data on the state of immunosuppression.

Another important finding was that just under twothirds of the adolescents have been fully disclosed of their HIV status. Surprisingly depression was not associated with disclosure in our univariate analysis (OR 1.34; $P=0.3$ ). There is a need for further studies to validate this observation. Health care workers may be deferring disclosure to ALHIV to those who are overtly depressed to manage their mental health first before a proper disclosure is planned. Disclosure we know is also a process which needs pre-disclosure, disclose, and post-disclosure stage planning [25]. One of the key steps for ALHIV is to gradually take on responsibility for one's own care. Full disclosure would then give meaning to the health education and actions that the adolescent is instructed to follow. The important questions before us are as follows: does disclosure trigger or worsen depression, and further to this does it have an impact on drug adherence if the disclosure experiences have been negative or ambivalent?

Although not associated with depression, just over one in 10 adolescents had made a sexual debut. Among those adolescents interviewed who were 12 years and older, $13.6 \%$ reported being sexually active or having had a sexual encounter. These experiences were more common among ages 15-19 years. According to Kenya Demographic and Health Survey 2014, 37.3\% of adolescents aged 15-19 years reported ever having sex [26] a higher prevalence compared to our study participants suggesting that HIV-infected adolescents may be postponing sexual debut or have delays in development due to recurring or chronic illness. There is considerable variability in proportion of HIV-infected adolescents who have achieved sexual debut with a Ugandan study reporting a third [15], while in South Africa, Toska et al. [27] found a prevalence of $14.9 \%$ in their study sample. These differences may reflect variability in the age composition of the study population and also the prevalent norms on adolescent sexuality in different cultural settings.

In our study, the majority of the adolescents who had achieved sexual debut reported inconsistent condom use similar to a Ugandan study that showed $76.5 \%$ of ALHIV inconsistently used condoms [9], a finding that underscores the need to bolster sexual and reproductive health education in this group. Inconsistent use of condoms poses important challenges including reinfection of a HIV-infected partner with risk of transmitting resistant strains, transmission of HIV to their uninfected partners, and acquisition and transmission of sexually transmitted diseases. In our study, $27 \%$ of adolescents did not think that condoms reduced HIV transmission, while another 26.3\% did not know if condoms reduced transmission of HIV. This is also an alarming response from our participants whose knowledge about sexual protection appeared to be deficient warranting a better framing of SRH education policy in schools and communities. The majority of adolescents in our study obtained condoms from health facilities; however, health care workers did not feature as a key source of information about HIV and other reproductive health issues. This maybe an indication of limited contact between adolescents and health workers or missed opportunities within the health facilities or lack of 
a structured method for collecting information like this study did using the HEADSS framework.

We think that the HEADSS framework offered us an opportunity to identify the adolescents who needed more immediate or focused services and further management. An alarming finding in this context was that one in four sexual debuts might have been due to a forced encounter or due to abuse. This clearly conveys the evidence of adverse childhood experiences that need to be understood better and adolescents who have had an adverse experience of coerced sex or abuse need emergency services, counseling, and socio-legal protection from further abuse. The adolescent clinic can be the point of referral for such young persons to child protection services who would need to be more responsive to needs of those young people who present with chronic illnesses or infections like HIV. Another notable finding is that about nine out of ten ALHIV are not sexually active and this offers a great opportunity to introduce psychoeducation and preventive health services that such adolescents could benefit from. Building their parents/guardians' or health worker skills on how to support their adolescent to adopt safe approach to their sexuality would be the next step.

This study has several strengths that include a validated adolescent evaluation framework such as HEADSS. The study was conducted in a well-established clinic that had followed many of the adolescents since childhood in the context of their HIV care. All the adolescents were on antiretroviral drugs. The study was conducted during the school holidays so that adolescents in boarding school had an equal opportunity to participate similar to those in day schools. All the adolescents approached for the study accepted to participate and so did their guardians indicating that there is minimal non-participation bias and eagerness to share experiences in this group. In order to ensure informed consent and respect for the relationship between the adolescent and the parent/guardian, the researchers first approached the guardian for permission before assent by the adolescent. This overwhelming participation probably reflects that young people are very responsive to mental health care services and do have a strong desire to engage with providers on addressing their needs.

In published literature, the PHQ-9 tool has been validated among non-HIV-infected adolescents in the USA [16]. In Africa, this tool has been used among HIV-infected adults in Western Kenya [20] and among adolescents living with HIV in Tanzania. In the latter study, severity of depression was computed as score of 10 or greater based on the validation studies done in adults in Western Kenya [12]. We are therefore reasonably confident that our estimates of depression in this study are valid. The adapted HEADSS tool enabled the identification of 12 adolescents with suicidal ideation. This was $4 \%$ of the study population and was the first time this diagnosis was made in these adolescents. This finding alone is a strong justification for routine use of HEADSS tool in assessing HIV-infected adolescents.

There is a need to delve further and make a DSM-V diagnosis of depression as part of the strategy to develop more refined criteria for diagnosis of major depression among ALHIV. This study contributes to the growing body of studies using standardized tools. There is a need for validation of this abbreviated tool against the comprehensive HEADSS and other measures of depression such as Beck Depression Inventory version II (BDI-II) and The Children's Depression Rating Scale-Revised (CDRS-R).

A major limitation of the study was its cross-sectional design, meaning that exposure and outcome were measured at the same time, and therefore causal relationships could not be ascertained. Further to this, the study did not use the full-length HEADSS tool and therefore there is a need for further work to adapt the tool to our environment. Many of the questions posed in this tool are sensitive and there may have been some bias if adolescents engaged in face-to-face interviews under reporting or gave socially acceptable responses. Despite these limitations, the interviews tapped into important areas which need to be investigated in further details. The study did not have a comparison group of adolescents with a different chronic illness and therefore cannot establish whether the depression symptoms are unique to HIVinfected adolescents or a feature of adolescents struggling with chronic illness.

\section{Conclusion}

Overall, our work highlights the need to tap into depression, school performance, adherence and engagementrelated barriers and challenges, as well as sexual and reproductive health awareness needs. One in two ALHIV in our study had depression symptoms. HEADSS and PHQ-9 tools should be incorporated into routine care of adolescents. Further studies need to be conducted to determine causal relationship of the factors identified as the predictors of depression.

\footnotetext{
Abbreviations

ALHIV: adolescents living with HIV; HEADSS: home environment, education and employment, activity, sexuality, suicide and depression traits; PHQ-9:

Patient Health Questionnaire; MHP: mental health problem; SRH: sexual and reproductive health; CARV: combined antiretroviral; APOC: adolescent package of care.

Authors' contributions

DG, RN, DW, and MK designed the study. Data collection was carried out by DG, NW, and MK. Data analysis and writing of the paper was done by DG, RN, MK, and DW. All authors read and approved the final manuscript.
} 


\section{Author details}

${ }^{1}$ Department of Paediatrics, College of Health Sciences, University of Nairobi, P.O. Box 19676, Nairobi, Kenya. ${ }^{2}$ Department of Psychiatry, College of Health Sciences, University of Nairobi, P.O. Box 19676, Nairobi, Kenya.

\section{Acknowledgements}

We thank the adolescents for participating in the study and the staff at Kenyatta National Hospital Comprehensive care center. Thank you to Dr. Rachel vreeman for her valuable input.

\section{Competing interests}

The authors declare that there are no competing interests.

\section{Availability of data and materials}

The data set used and/or analyzed during the current study is available from the corresponding author on reasonable request.

\section{Consent for publication}

Participants consented to study findings being published in scientific journals and conferences.

\section{Ethics approval and consent to participate}

Ethical approval was obtained from The Ethics and Research Committee of Kenyatta National Hospital and University of Nairobi (approval no. P217/03/2016). All 270 participants who were approached consented to participate.

\section{Funding}

Collection of data was supported by a grant to the first author from AMPATH (Academic Model Providing Access To Healthcare) which is part of a scholarship to pursue Masters of Medicine in Paediatrics and Child Health at the University of Nairobi.

\section{Publisher's Note}

Springer Nature remains neutral with regard to jurisdictional claims in published maps and institutional affiliations.

Received: 14 May 2018 Accepted: 29 June 2018

Published online: 10 July 2018

\section{References}

1. UNAIDS. Global report: UNAIDS report on the global AIDS epidemic 2013 [Internet]. Unaids. 2013. p. 198. www.unaids.org/.../unaids/.../2013/ gr2013/UNAIDS Global_Report 2013. Accessed 11 Dec 2017.

2. UNAIDS/UNICEF. All Into \#EndAdolescentAIDS. 2015;95.

3. National AIDS Control Council. Kenya's fast-track plan to end HIV and AIDS among adolescents and young people. 2015;(September):40.

4. World Health Organization. Global accelerated action for the health of adolescents (AA-HA!). 2017. http://apps.who.int/iris/bitstream/handl e/10665/255415/9789241512343-eng.pdf; jsessionid=B2626A5AB85675D 96B840C78270E4955?sequence=1. Accessed 11 Dec 2017

5. Folayan MO, Odetoyinbo M, Harrison A, Brown B, Trinity H, Plaza H, et al. Addressing the socio-development needs of adolescents living with HIV/ AIDS in Nigeria: a call for action. Afr J Reprod Health. 2015;18:93-101.

6. Scorza P, Kanyanganzi F, Smith MC, Sezibera V, Cyamatare F, Beardslee W, et al. HIV and child mental health : a case-control study in Rwanda. Pediatrics. 2014. https://doi.org/10.1542/peds.2013-2734.

7. Louw K, Ipser J, Phillips N, Hoare J. Correlates of emotional and behavioural problems in children with perinatally acquired HIV in Cape Town, South Africa. AIDS Care. 2016. https://doi.org/10.1080/09540 121.2016.1140892.

8. Lowenthal ED, Marukutira TC, Chapman J, Mokete K, Riva K, Tshume O, et al. Psychosocial assessments for HIV+ African adolescents : establishing construct validity and exploring under-appreciated correlates of adherence. PloS ONE. 2014. https://doi.org/10.1371/journal.pone.01093 02
9. Mutumba M, Bauermeister A. Psychosocial challenges and strategies for coping with HIV among adolescents in Uganda. AIDS Patient Care STDS. 2015:29(2):86-94.

10. Souza E, Santos N, Valentini S, Silva G, Falbo A. Long-term follow-up outcomes of perinatally HIV-infected adolescents: infection control but school failure. J Trop Pediatr. 2010;56(56):421-6.

11. Enimil A, Nugent N, Amoah C, Norman B, Ocran J, Kwara A, et al. Quality of life among Ghanaian adolescents living with perinatally acquired HIV : a mixed methods study. AIDS Care. 2015. https://doi.org/10.1080/09540 121.2015.1114997.

12. Dow DE, Turner EL, Shayo AM, Mmbaga B, Cunningham CK, Donnell $\mathrm{KO}$, et al. Evaluating mental health difficulties and associated outcomes among HIV-positive adolescents in Tanzania. AIDS Care. 2016. https://doi. org/10.1080/09540121.2016.1139043.

13. Nabukeera-barungi N, Elyanu P, Asire B, Katureebe C, Lukabwe I, Namusoke $\mathrm{E}$, et al. Adherence to antiretroviral therapy and retention in care for adolescents living with HIV from 10 districts in Uganda. BMC Infect Dis. 2015. https://doi.org/10.1186/s12879-015-1265-5.

14. Kim MH, Mazenga AC, Yu X, Devandra A, Nguyen C, Ahmed S, et al. Factors associated with depression among adolescents living with HIV in Malawi. BMC Psychiatry. 2015;15:264. http://www.pubmedcentral.nih. gov/articlerender.fcgi?artid $=4624356 \&$ tool=pmcentrez\&rendertype =abstract. Accessed 11 Dec 2017.

15. Mbalinda SN, Kiwanuka N, Eriksson LE, Wanyenze RK, Kaye DK. Correlates of ever had sex among perinatally HIV-infected adolescents in Uganda. Reprod Health. 2015. https://doi.org/10.1186/s12978-015-0082-z.

16. Cappelli AM, Gray C. The HEADS-ED: a rapid mental health screening tool for pediatric patients in the emergency department. Pediatrics. 2012. https://doi.org/10.1542/peds.2011-3798.

17. Ministry of Health. Guidelines on use of antiretroviral drugs for treating and preventing HIV infection in Kenya. 2016

18. National Adolescent Sexual and Reproductive Health Policy, Ministry of Health, Kenya 2015; http://aphrc.org/wp-content/uploads/2015/09/Minis try-of-Health-ASRH-Booklet-Final-1.pdf. Accessed 11 Dec 2017.

19. Kenya Vision 2030. A globally competitive and prosperous Kenya. 2007 https://www.researchictafrica.net/countries/kenya/Kenya_Vision_2030__2007.pdf.

20. Shavisa T, Ndiku J, Musasia M. The role of student characteristics in dropout cases among secondary school students in Vihiga county, Kenya. 2016. p. 85-100.

21. Our future: a lancet commission on adolescent health and wellbeing: the lancet, 2013 https://www.thelancet.com/pb/assets/raw/Lancet/stories/ commissions/. Accessed 11 Dec 2017.

22. Kenya Reproductive, Maternal, Newborn, Child and Adolescent Health (RMNCAH) investment framework; Ministry of Health Government of Kenya January 31, 2016

23. Vreeman RC, Nyandiko WM, Liu H, Tu W, Scanlon ML, Slaven JE, et al. Measuring adherence to antiretroviral therapy in children and adolescents in western Kenya. J Int AIDS Soc. 2014;1-10:19227.

24. Dolezal C, Mckay M, Wiznia A. Mental health and family influences. 2011

25. Montalto G, Sawe F, Miruka A, et al. Diagnosis disclosure to adolescents living with HIV in rural Kenya improves antiretroviral therapy adherence and immunologic outcomes: a retrospective cohort study. PloS ONE. 2017. https://doi.org/10.1371/journal.pone.0183180.

26. Kenya National Bureau of Statistics (KNBS) and ICF Macro. Kenya demographic and health survey 2008-09. Calverton: KNBS and ICF Macro; 2010

27. Toska E, Cluver LD, Hodes R, Kidia KK. Sex and secrecy: how HIV-status disclosure affects safe sex among HIV-positive adolescents. AIDS Care. 2015;27:47-58 